\title{
Pediatric Pulmonary Tuberculoma with a Solid Pulmonary Nodule Detected on Chest Computed Tomography
}

\author{
Atsuhito Ushiki ${ }^{1}$, Yoshitaka Yamazaki ${ }^{2}$, Gen Ideura $^{3}$, Takashi Shinbo ${ }^{2}$, \\ Mariko Sugawara ${ }^{2}$, Mineyuki Hama ${ }^{1}$ and Masayuki Hanaoka ${ }^{1}$
}

\begin{abstract}
A 14-year-old girl underwent a medical checkup for Mycobacterium tuberculosis infection because her grandmother had been diagnosed with pulmonary tuberculosis three months earlier. The interferon-gamma release assay (IGRA) showed a positive result. The patient's chest X-ray findings were normal. Chest computed tomography (CT) showed a single mass lesion in the right lower lobe of the lung. A sputum smear of acid-fast bacilli was positive; however, the polymerase chain reaction results for tuberculosis were negative. We diagnosed the patient with pulmonary tuberculosis based on the fact that she had come in contact with a tuberculosis patient. Six weeks later, a liquid culture examination for acid-fast bacilli was found to be positive and the acid-fast bacillus was identified as M. tuberculosis. The use of chest CT is not routinely recommended in all children suspected of having M. tuberculosis infection. However, IGRA-positive children who report frequent contact with infected individuals should undergo CT tomography if chest X-rays do not show any abnormal shadows.
\end{abstract}

Key words: pulmonary tuberculosis, latent tuberculosis infection, interferon-gamma release assay, chest computed tomography

(Intern Med 53: 913-916, 2014)

(DOI: 10.2169/internalmedicine.53.1880)

\section{Introduction}

Today, one-third of the world's population is infected with Mycobacterium tuberculosis, and pulmonary tuberculosis is one of the most serious public health problems in both developing and developed countries (1). In Japan, the incidence of tuberculosis fell below 20 per 100,000 individuals in 2007 and continued to decline, reaching a rate of 18.2 in 2010. However, 23,261 tuberculosis patents were newly identified in 2010 (2). Recently, the number of patients diagnosed with diabetes mellitus or human immunodeficiency virus and those receiving treatment with hemodialysis tumor necrosis factor- $\alpha$ inhibitors has increased. Such patients are at high risk of tuberculosis infection. The number of new pediatric tuberculosis cases in Japan fell to below 100 in 2006 (2). Of the 73 pediatric tuberculosis patients newly identified in 2009, $33(45.2 \%)$ were identified at medical in- stitutions with some signs or symptoms and 24 (32.9\%) were identified based on the results of examinations in individuals with a history of family contact (3).

The general procedures used for contact examinations include the tuberculin skin test (TST) and interferon gamma releasing assay (IGRA). A chest X-ray is obtained detect active pulmonary tuberculosis in cases involving positive results on TST or IGRA or patients with symptoms. Patients diagnosed with active tuberculosis receive multi-drug treatment, while those diagnosed with latent tuberculosis infection (LTBI) receive isoniazid or rifampicin monotherapy (4). Patients with active tuberculosis should not be treated with monotherapy because monotherapy can induce resistant bacilli strains.

We herein report a case of active pulmonary tuberculosis diagnosed in a 14-year-old girl who was first diagnosed with LTBI based on the results of IGRA and a chest X-ray.

${ }^{1}$ The First Department of of Internal Medicine, Shinshu University School of Medicine, Japan, ${ }^{2}$ Department of Infectious Disease, Suzaka Hospital, Japan and ${ }^{3}$ Department of Respiratory Medicine, Shinshu Ueda Medical Center, Japan

Received for publication October 7, 2013; Accepted for publication November 21, 2013

Correspondence to Dr. Yoshitaka Yamazaki, yamazaki-yoshitaka@pref-nagano-hosp.jp 


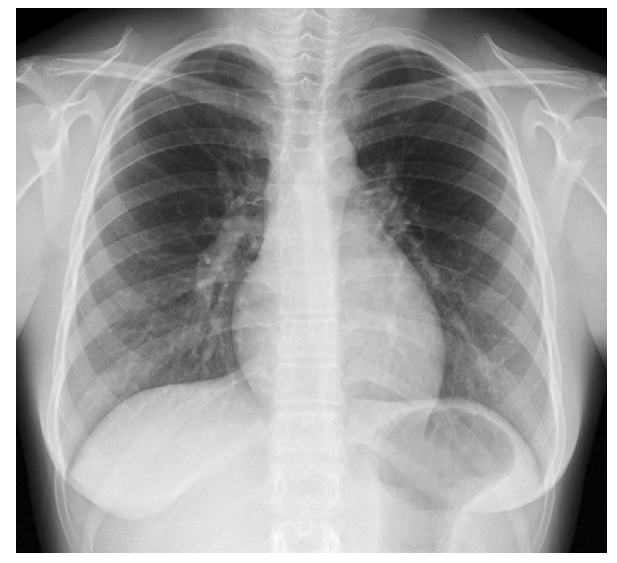

Figure 1. Chest X-ray obtained on admission. No abnormal shadows were detected.

\section{Case Report}

A 14-year-old girl underwent a medical checkup for $M$. tuberculosis infection at the hospital in July 2012. Three months previously, her grandmother had received a diagnosis of pulmonary tuberculosis (Gaffky 6 in the grandmother's sputum). The patient was suspected of having LTBI based on a positive QuantiFERON ${ }^{\circledR}$ TB-3G (QFT) result, with an interferon-gamma (IFN- $\gamma$ ) concentration of more than $10 \mathrm{IU} / \mathrm{mL}$. She had no symptoms or unusual medical history. The findings of the physical examination were normal.

The patient's laboratory data showed a normal white blood cell count and normal C-reactive protein level (Table). A chest X-ray did not show any abnormal shadows (Fig. 1). Chest computed tomography (CT), however, revealed a mass lesion surrounded by ground grass opacity in the right S10 (Fig. 2A). A smear of acid-fast bacilli obtained from the patient's white sputum was positive in only one of three consecutive expectorated sputum samples. Meanwhile, a polymerase chain reaction (PCR) study for tuberculosis was negative (Table). The chest CT findings suggested tuberculoma, a tumor or organizing pneumonia.

We performed bronchoscopy to confirm the diagnosis. No atypical cells or acid-fast bacilli were detected in the bronchial washing fluid. We therefore diagnosed the patient with pulmonary tuberculosis based on her frequent contact with an infected person and the sputum smear findings and initiated treatment with isoniazid, rifampicin, ethambutol and pyrazinamide for six months. Liquid culture examinations of acid-fast bacilli obtained from the sputum were positive six weeks later, and the acid fast bacillus isolated from the cultured colony was identified to be M. tuberculosis on PCR (Table). After six months of treatment with antituberculosis drugs, chest CT showed only a small scar in the right lower lobe of the lung (Fig. 2B). We instructed the patient not to attend school until the culture examinations of acid-fast bacilli from her sputum were negative on three consecutive oc- casions.

\section{Discussion}

We herein reported a case of pediatric pulmonary tuberculoma. A smear and culture of acid-fast bacilli was positive in only one of three consecutive expectorated sputum and bronchial washing samples. In the present case, the expectorated sputum was white, similar to saliva. Detecting the lesion on chest X-ray was difficult using bronchoscopy, because the lesion was located behind the cardiac shadow. Therefore, sputum and bronchial washing samples were not accurately collected from the lesion.

The QFT is a high sensitivity and specificity test used to diagnose tuberculosis infection. The sensitivity and specificity of QFT in adult patients with active pulmonary tuberculosis is $89.0 \%$ and $98.1 \%$, respectively (5). Detjen et al. reported the usefulness of QFT in diagnosing pediatric pulmonary tuberculosis. They assessed the use of QFT in 28 children with bacteriologically-confirmed tuberculosis (27 cases of pulmonary disease and one case of extrapulmonary disease) and found the sensitivity and specificity of QFT in pediatric patients with active tuberculosis infection to be $93.0 \%$ and $100 \%$, respectively (6). The QFT is an important test, especially in cases of pediatric pulmonary tuberculosis, because it is difficult to detect M. tuberculosis using smears, culture and PCR. Nevertheless, the sensitivity of QFT is lower than that of TST in children with LTBI, especially infants (7), for several reasons. First, IFN- $\gamma$ production is induced by various antigens in infants (8). Furthermore, the amount of tuberculosis in patients with LTBI is lower than that in cases of active pulmonary tuberculosis. Therefore, the rate of stimulation of tuberculosis antigens is low, and IFN- $\gamma$ production is not induced. However, the mitogen control IFN- $\gamma$ responses in schoolchildren are higher than those observed in infants (7); therefore, the QFT is useful for diagnosing LTBI in schoolchildren.

The present patient was first suspected to have LTBI because she had no respiratory symptoms and her chest X-ray showed no abnormal shadows. The use of chest CT is not routinely recommended in all children suspected of having LTBI due to the high level of exposure to radiation, high cost and need for sedation. However, cases of pediatric pulmonary tuberculosis almost always involve primary tuberculosis, and primary tuberculosis lesions are small (9). Chest X-rays may fail to detect these small lesions (10). QFTpositive children with respiratory symptoms or frequent contact with infected individuals, as in the present case, should therefore undergo chest CT if chest X-rays do not show any abnormal shadows.

The chest CT scan in the present case revealed a solid nodule in the right S10. Secondary tuberculosis lesions are often formed in S1, S2 and S10. On the other hand, primary tuberculosis lesions, as observed in the present case, can form anywhere in the lungs. Kim et al. investigated the chest CT findings of 41 children with pulmonary tuberculo- 

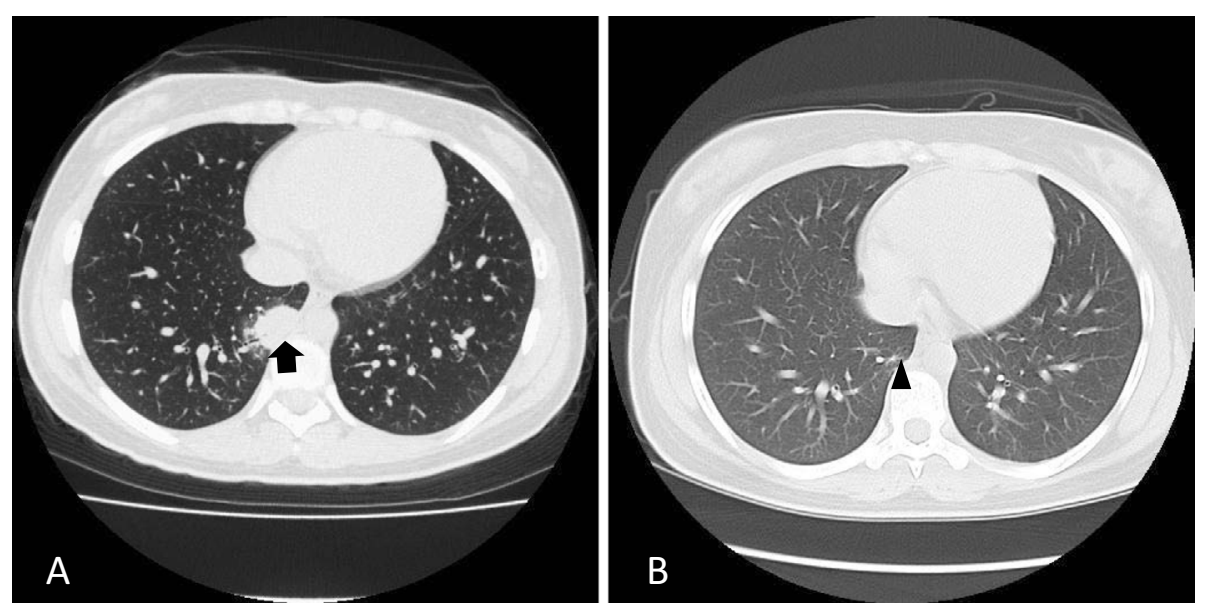

Figure 2. A: Chest computed tomography (CT) performed before treatment. CT showed a mass lesion that was difficult to visualize on a chest $\mathrm{X}$-ray, with an irregular border measuring $3 \mathrm{~cm}$ in diameter in the right S10 (arrow). The lesion was surrounded by ground grass opacity. B: Chest CT after treatment. CT showed only an inflammatory scar in S10 (arrowhead).

Table. Laboratory Data

\begin{tabular}{|c|c|c|c|}
\hline \multicolumn{3}{|c|}{ Hematology } & \multirow{2}{*}{$\frac{\text { Serology }}{\text { CRP } 0.10}$} \\
\hline WBC & 5,400 & $/ \mu \mathrm{L}$ & \\
\hline $\mathrm{RBC}$ & $458 \times 10^{4}$ & $/ \mu \mathrm{L}$ & \\
\hline $\mathrm{Hb}$ & 13.5 & $\mathrm{~g} / \mathrm{dL}$ & $\underline{\text { IGRA }}$ \\
\hline Plt & $27.2 \times 10^{4}$ & $/ \mu \mathrm{L}$ & QuantiFERON® TB-3G \\
\hline \multicolumn{4}{|c|}{ Biochemistry } \\
\hline Alb & 3.7 & $\mathrm{~g} / \mathrm{dL}$ & Acid-fast Bacilli in Sputum \\
\hline AST & 12 & $\mathrm{IU} / \mathrm{L}$ & Day 1 smear Gaffky 1, PCR of tuberculosis (-), \\
\hline ALT & 8 & $\mathrm{IU} / \mathrm{L}$ & culture $(+)$ \\
\hline $\mathrm{LDH}$ & 147 & $\mathrm{IU} / \mathrm{L}$ & $\rightarrow$ identified as M.tuberculosis by PCR \\
\hline ALP & 250 & $\mathrm{IU} / \mathrm{L}$ & Day 2 smear $(-)$, culture $(-)$ \\
\hline $\mathrm{ChE}$ & 210 & $\mathrm{IU} / \mathrm{L}$ & Day 3 smear (-), culture (-) \\
\hline$\Gamma-\mathrm{GTP}$ & 6 & $\mathrm{IU} / \mathrm{L}$ & \\
\hline T.Bil & 0.4 & $\mathrm{mg} / \mathrm{dL}$ & Acid-fast Bacilli in Bronchial Washing Fluid \\
\hline Amy & 61 & IU/L & $\overline{\text { Smear }(-), \text { culture }(-)}$ \\
\hline BUN & 10.4 & $\mathrm{mg} / \mathrm{dL}$ & \\
\hline $\mathrm{Cr}$ & 0.56 & $\mathrm{mg} / \mathrm{dL}$ & \\
\hline $\mathrm{Na}$ & 141 & $\mathrm{mEq} / \mathrm{L}$ & \\
\hline K & 4.0 & $\mathrm{mEq} / \mathrm{L}$ & \\
\hline $\mathrm{Cl}$ & 107 & $\mathrm{mEq} / \mathrm{L}$ & \\
\hline Glu & 83 & $\mathrm{mg} / \mathrm{dL}$ & \\
\hline
\end{tabular}

IGRA: interferon-gamma releasing assay, PCR: polymerase chain reaction

sis and reported mediastinal and hilar lymphadenopathy to be the most frequent finding, noted in 34 patients, followed by air space consolidation and nodules of bronchogenic spread (20 and 12 patients, respectively). Furthermore, there were no cases in which chest CT showed solid nodules (9). Curtis et al. also reported that solid nodules constitute a very infrequent form of pulmonary tuberculosis in children (11). On the other hand, M. tuberculosis occasionally results in the formation of solitary nodules in adults (12). This type of solid nodule is termed tuberculoma. Pathologically, tuberculomas are caseous masses encapsulated by multiple concentric layers of connective tissue. The proposed mechanism underlying the development of tuberculomas consists of repeated extension of bronchopneumonic foci, subsequent necrosis and re-encapsulation (13). Shrinkage of occluded cavities and fusion of small areas of encap- sulated density have been suggested as other mechanisms (13). Tuberculomas are thought to be the result of a protective mechanism by the host's immune system against the tuberculosis infection. Because the immune system is immature in children, tuberculomas are rare. However, in schoolchildren, the immune system is more mature, and tuberculomas are occasionally detected.

In conclusion, IGRA-positive children who report frequent contact with sick people, especially those with respiratory symptoms, should undergo chest CT because chest Xrays may fail to detect small lesions. Chest CT occasionally shows single nodules in children with pulmonary tuberculosis, especially school-aged children.

The authors state that they have no Conflict of Interest (COI).

\section{References}

1. JI B, Lounis N, Truffot-Pernot C, Grosset J. In vitro and in vivo activities of levofloxacin against Mycobacterium tuberculosis. Antimicrob Agents Chemother 39: 1341-1344, 1995.

2. Tuberculosis Surveillance Center, RIT, JATA. Tuberculosis annual report 2010: (1) summary of tuberculosis notification statistics in 2010. Kekkaku 87: 481-485, 2012 (in Japanese, Abstract in English).

3. Center Tuberculosis Surveillance Center, RIT, JATA. Tuberculosis annual report 2009: series 3. childhood TB. Kekkaku 86: 553-556, 2011 (in Japanese, Abstract in English).

4. Targeted tuberculin testing and treatment of latent tuberculosis infection. This official statement of the American Thoracic Society was adopted by the ATS Board of Directors, July 1999. This is a Joint Statement of the American Thoracic Society (ATS) and the Centers for Disease Control and Prevention (CDC). This statement was endorsed by the Council of the Infectious Diseases Society of America (IDSA), September 1999, and the sections of this statement. Am J Respir Crit Care Med 161: S221-S247, 2000.

5. Mori T, Sakatani M, Yamagishi F, et al. Specific detection of tuberculosis infection: an interferon- $\gamma$-based assay using new antigens. Am J Respir Crit Care Med 170: 59-64, 2004.

6. Detjen AK, Keil T, Roll S, et al. Interferon- $\gamma$ release assays im- 
prove the diagnosis of tuberculosis and nontuberculous mycobacterial disease in children in a country with a low incidence of tuberculosis. Clin Infect Dis 45: 322-328, 2007.

7. Connell TG, Curtis N, Ranganathan SC, Buttery JP. Performance of a whole blood interferon gamma assay for detecting latent infection with Mycobacterium tuberculosis in children. Thorax 61: 616-620, 2006.

8. Smart JM, Kemp AS. Ontogeny of T-helper 1 and T-helper 2 cytokine production in childhood. Pediatr Allergy Immunol 12: 181187, 2001.

9. Kim WS, Moon WK, Kim IO, et al. Pulmonary tuberculosis in children: evaluation with CT. AJR Am J Roentgenol 168: 10051009, 1997.
10. Hirama T, Hagiwara $K$, Kanazawa M. Tuberculosis screening programme using the QuantiFERON-TB Gold test and chest computed tomography for healthcare workers accidentally exposed to patients with tuberculosis. J Hosp Infect 77: 257-262, 2011.

11. Curtis AB, Ridzon R, Vogel R, et al. Extensive transmission of Mycobacterium tuberculosis from a child. N Engl J Med 341: 1491-1495, 1999.

12. Hahm CR, Park HY, Jeon $\mathrm{K}$, et al. Solitary pulmonary nodules caused by Mycobacterium tuberculosis and Mycobacterium avium complex. Lung 188: 25-31, 2010.

13. Lee HS, Oh JY, Lee JH, et al. Response of pulmonary tuberculomas to anti-tuberculous treatment. Eur Respir J 23: 452-455, 2004.

(C) 2014 The Japanese Society of Internal Medicine http://www.naika.or.jp/imonline/index.html 